\title{
Preview or Not: An Innovation by the PAD Class Paradigm in English Extended Courses
}

\author{
CHEN Yingxi \\ Guangdong University of Foreign Studies South China Business College, Guangzhou, China \\ tracycici@qq.com
}

Keywords: Preview, PAD Class, Course Design, English Extended Courses.

\begin{abstract}
PAD Class paradigm (Presentation - Assimilation - Discussion) is an innovated teaching pattern, in which preview process is not encouraged. Based on this teaching pattern and the actual teaching situations, this paper aims to carry out a study of this reform and practice concerning with the preview activities for the university English extended courses. In this paper, PAD Class teaching paradigm, PAD Class teaching principles, and preview activities among other teaching methods have been reviewed, then class designs without preview activities for different university English extended courses have been introduced, so as to discuss that preview is not important in teaching and learning activities, which is an innovation in class teaching patterns.
\end{abstract}

\section{Introduction}

Since the reform and opening up policy was proposed and implemented in the 1980s, teaching methods have been gradually changing to adapt the more and more advanced society. For most of these modes, learning before teaching has been encouraged. In these teaching and learning patterns, students need to preview the lessons or the materials mostly provided by the teachers before they attend the class. Many schools got successful experiences from this way of teaching, however, there were many problems exposed in this learning process [1]. Under this concept, self-learning ability becomes a very important influential factor which varies among students. With the continuous development of the teaching modes, the PAD Class paradigm is to innovate. This teaching and learning pattern is without the preview process emphasized by other teaching methods. In this part, PAD Class teaching paradigm and principles, as well as the preview activities would be reviewed.

\subsection{PAD Class Teaching Paradigm}

Just as its name implies, the PAD Class teaching paradigm can be divided into three parts presentation part by the teacher, assimilation part by the students, and the discussion part within the study group and the whole class involving the teacher. This newly teaching paradigm is actually a combination of traditional lecturing teaching paradigm and a newer discussion one. In the procedures of PAD Class paradigm, assimilation part can be left to the students after class or in class, which results in cross-class PAD or in-class PAD. Compared the cross-class PAD with the in-class one, the former provides more assimilation time for the students. Thus, it is more welcomed by both the teacher and the students. In this case, a class is usually bisects into two parts - the students' discussions of the previous lesson and the teacher's presentation of the new lesson.

Teachers usually spend half of the class time doing presentation of the main points and difficult points of the chapter, leaving blanks for the students to assimilate later. For the other half part of the class, usually taken place before the teacher's presentation, students discuss the problems or findings from their self study within groups, followed by an interactive discussion among the teacher and the students. Actually, group discussion appeals to many other teaching paradigms and it is good for either the teacher or the student. However, the ability of the students varied. Not all of them know what to discuss in class, especially in an English class. In such a case, a class in silence or in Chinese discussions can usually be found. Hence, the effect of the discussion pattern does not always meet the teacher's expectation. This new teaching paradigm can fix the problem in a good way, for the 
students have already finished their assignment after class and what they could share with each other are their homework and findings. New ideas or questions might come up during their discussions.

This kind of teaching paradigm fits almost every subject. Since its first proposal in 2014, it has already gained national popularity in China and has successfully put into practice by thousands of college teachers in different grades of different subjects. The success of the PAD Class pattern relies on the base of psychological and pedagogical rationales to explicate its effectiveness in enhancing active learning. In the usual discussion classroom, the students are asked to do discussions right after teacher's lecture, which violates the basic psychological principles of learning.

\subsection{PAD Class Teaching Principles}

One needs time to think and assimilate the knowledge before he/she actually acquire the knowledge. Otherwise, the afterward discussion is lack of depth. Usually, when discussion is used in the class, it is often implemented in the form of instant discussion, which means that the students discuss right after the teacher's presentation. Although this pattern is also used in the in-class PAD, the task is often not very difficult, because this way of operation violates the basic psychological principles of learning [2].

In the psychological aspect of learning, students could or may not understand the study content they have just learned from the teacher. And, in class there is not enough time for them to think deep and assimilate the knowledge so they might have difficulties to raise questions. It would be very hard for them to have their own specific opinions. According to a anonymous survey in the author's class, asking for what they have learned in the previous class discussions, the students said that in the most of their previous discussions, they did not know what to say but for the interesting topics, they might be able to talk more but only when it was fun and not difficult. But study is not always a fun thing. However, if the study content is not very difficult for the students and the course curriculum is not allowed a cross-class PAD pattern, then the quick PAD pattern, an in-class PAD can be used.

In PAD Class paradigm, the point is to modify "instant discussion" to "delayed discussion" [3]. The key to the procedure of cross-class PAD discussions is to assimilate first. Then, doing homework and review the lessons are important to successfully carry out this paradigm. Here, a question is raised: whether it is needed to preview the lessons.

\subsection{Preview Activities in Teaching Process}

In the PAD Class paradigm, the teacher should not teach too much because if the teacher presents too clear and too profound, the students would not have the space and time to assimilate and to think on their own. In this case, do the students need to preview the lessons? In general, preview is important. Almost in the recent 20 or even more years of teaching and learning, preview activity is always encouraged by the teachers and parents. Although teaching methods have been gradually introduced to the classroom, especially with the help of the internet, for example, Mooc, flipped classroom, etc., it is still a valued part to preview lessons. Moreover, in some newly teaching methods, if the students could not study beforehand, it would be impossible for them to catch what the teacher presents in class.

The essence of presentation in PAD Class is to concise teaching and to leave blank. In this way, students are required to do autonomous learning. However, it does not mean that the students need to preview the lessons even when they need to do the self-study. Instead, preview is not encouraged, because it brings quite a lot of problems also. In the most of the teaching patterns with preview activity, the main point is to teach after learning, which means that the students need to first learn the new contents. However, in such a learning process, the individual differences among different levels of students are artificially widened. Some tasks might be or must be assigned to "help" the students tackle the preview activity. After that, some students might think that they have already "learned" the content so that they might have no interests in listening to the teacher in class. In this case, it would be impossible to continue an inquiry learning [4].

From the psychological perspective, whenever the students meet the difficulties during the process of preview, they would come across a psychological dilemma in decision [5]. If they give up, 
they cannot complete the preview task; if not, they might spend quite a lot of time working on it with a not satisfied result. In both cases, they would be frustrated. If there might be some opportunities to bring greater psychological burdens to the students, then why is it needed? Besides, preview reduces students' curiosities to concentrate themselves in class, which is definitely not a good way for the teaching and learning activities. Needless to say, it is a great innovation for class paradigm to have no preview activity.

\section{Class Design for Extended English Courses without Preview Activity}

Nowadays, students have various extended English courses to choose. There are mainly two aspects of courses: English for special purpose (ESP), and cultural type English (CTE). It might be of great differences among these two types of courses but the class designs using PAD Class paradigm are quite similar. In the following part, class designs without preview activity of two courses would be analyzed, Tourism English [6] for the ESP class, and American society and culture [7] for the CTE class.

It would be a very interesting thing to compare these two courses because they have a huge difference in the understanding levels. For the ESP class, since it is English for specific academic purposes, the contents of this course are far more difficult than that of the American society and culture. If most of the students are still able to understand the basic knowledge of the textbook with the help of the internet resources or dictionaries, it is unlikely for them to comprehend the contents of the textbook of Tourism English even when they find out all the new words in the dictionary. Even when the author adopts the PAD Class paradigm, students still find it very difficult to review and comprehend all the contents of the textbook. However, most of them admit that they are now much clearer about the language skills mentioned either in the textbook or in the class because they fix their attention more in class.

Take the first unit of Tourism English for example, there are four parts in this unit: vocabulary (guessing words in context, prefixes and suffixes), listening (preparing for a lecture, predicting lecture content, making notes), extending skills (choosing the right kind of notes, making notes, speaking from notes) and a summary of vocabulary bank (guessing words in context) and skills bank (making perfect lecture notes, speaking from notes). The contents are not easy for the students so even when they try to preview the lesson, they would find it too difficult to comprehend. Then, it would be a waste of time to preview the book. In fact, one of the characteristics of this textbook is that the language skills mentioned in each unit would be repeated again and again to help the students to master the skills. In this case, it would be good for the teacher to present the skills first, perhaps with some examples from the textbook. After that, the students finish the assignment and think about the difficult points in the textbook for group discussions. If the problems cannot be solved within group, then a whole-class discussion with the teacher intervening would be held.

If Tourism English is so difficult for the students that it is of no use to do the preview, then there is another type of English courses - American society and culture. Take the first module for example, there are mainly two parts in this module: task one (a lead-in story of the American flag), and task two (four passages of four different topics related to fast facts about America). Of course, this unit also includes some other interesting extra-curricular parts like "person of the module" and "movie of the module" for the students to read after class, as well as another task of the guided writing or thinking. The contents are not difficult for the students to comprehend. Then, if the students do preview the lessons before, most of them would have no interests to keep on reading the textbook or listening to the teacher in class. However, if they do not preview the lessons, they should concentrate more on what the teacher has just mentioned, perhaps about the event, or about the sentence structure in the textbook and need to comprehend right away in class. In the presentation part, the teacher may use the passage in task one as a lead-in, by asking questions related to the passage. Then the outlines of the next four topics are listed as well as some important or difficult sentences are explained. After that, some videos relate to this module would be played so that the students might have a better understandings of the topics. Usually, the assignment would be the impressive things or important 
notes in the module. In the discussion part, students might orally sum up what they have done in their assignments or the problems they have found from the textbook, followed by a whole-class discussion.

\section{Summary}

The PAD Class paradigm is still quite new for Chinese classroom. It cannot be said that this paradigm is so perfect that it has no shortages. However, preview is a valued topic in Chinese classroom. In the recent related articles, strategies concerning the preview problems, learning methods of preview activities, and preview materials were studied [8], but no researches were made of this newly class paradigm, more importantly, without preview activities, which is an innovation to both the students and teacher.

\section{Acknowledgement}

This research was financially supported by the Key Program of the English Language and Literature of Guangdong Province, 2016; by the research of Guangdong Provincial Quality Project --- Teaching Research and Reform Project of Higher Education in Guangdong, 2017, "A Practical Research of the PAD Class Pattern in the College English Extended Courses"; and by the project "A PAD Class Paradigm Study for English Extended Courses under Educational Informatization Background" for Guangdong University of Foreign Studies South China Business College (Grant No. 2017JG02).

\section{References}

[1] G. Chen, Research of preview guiding under the mode of "learning before teaching" --- a case study of high school biology teaching, School of Education of Normal University, 2013.

[2] X. Zhang, The PAD Class, a new paradigm for university classroom teaching, $14^{\text {th }}$ Conference on Education and Training in Optics and Photonics: ETOP, 2017.

[3] X. Zhang, The PAD Class, a new exploration in university classroom teaching reform, Fudan Education Forum, vol.12, pp. 5-10, 2014.

[4] C. Li, A rational critique to "learning before teaching", People's Education, vol. 24, pp. 36-37, 2011.

[5] X. Zhang, PAD Class: new wisdom of Chinese education, Science Press, 2016.

[6] H. Mol, English for Tourism and Hospitality, Higher education press, 2016.

[7] H. Leng and G. Dong, American society and culture, Higher Education Press, 2014.

[8] J. Peng, Micro-video enhances preview of class's quality - promote research, Northwest Normal University, 2015. 